\title{
La práctica pedagógica de cinco egresados de Licenciatura en Química de la Universidad Distrital, implicada en la enseñanza de la Tabla Periódica ${ }^{1}$
}

\author{
María Victoria Ruiz Arandia 2 \\ mvruiza@udistrital.edu.co \\ Sol mercedes Castro Barbosa ${ }^{3}$ \\ solmer18@hotmail.com
}

Oscar Reyned Huertas Moya ${ }^{4}$

ohuertasm@udistrital.edu.co

\section{RESUMEN}

Este artículo es el resultado de la observación de las prácticas pedagógicas en torno al tema de la enseñanza de la tabla periódica, desarrolladas por cinco docentes en ejercicio, egresados de la licenciatura en química de la UD, en cuatro Instituciones Educativas Distritales. Este análisis gira en torno a las categorías: currículo, práctica pedagógica, competencia profesional, modelo pedagógico y tabla periódica, que buscan indagar, develar y generar la reflexión sobre el proceso de enseñanza en el aula de clase, con el fin de promover otros proyectos de investigación que conduzcan al fortalecimiento del proceso tanto de docentes en formación como de los egresados.

\footnotetext{
Este artículo hace parte del proyecto de investigación titulado «Diagnóstico de las prácticas pedagógicas de los egresados en ejercicio de la Licenciatura en Química de la Universidad Distrital Francisco José de Caldas", investigación financiada por el Centro de Investigaciones y Desarrollo Científico.

2 Ingeniera Química. Profesora asistente de la Facultad de Ciencias y Educación de la Universidad Distrital "Francisco José de Caldas"

3 Licenciada en Lingüística. Profesora titular de la Facultad de Ciencias y Educación de la Universidad Distrital "Francisco José de Caldas"

4 Bioquímico. Profesor asistente de la Facultad de Ciencias y Educación de la Universidad Distrital "Francisco José de Caldas"
} 
El diseño metodológico se fundamenta en la etnografía porque propicia la interpretación cultural al interior de las comunidades en interacción, permitiendo dilucidar los acontecimientos del proceso educativo, en donde el discurso tuvo un papel fundamental, ya que se indago acerca de la formación del egresado e identifico situaciones que cobraron significado en las prácticas pedagógicas.

\section{Palabras Clave:}

Currículo, Práctica pedagógica, competencia profesional, modelo pedagógico y tabla periódica.

\section{ABSTRACT.}

: This article is the result of the observation of the pedagogical practices about of the education of the periodic table, developed by five docent in exercise, UD professional chemist and four Educative institutions Distritales. This analysis turns around the categories: curriculum, pedagogical Practice, professional competition, pedagogical model and periodic Table that they look to investigate, to reveal and to generate the reflection on the education process in the classroom, with the . purpose of promoting other projects of investigation that lead as much to the fortification of teachers in formation process as the professionals.

The methodological design is based on the ethnography which causes the cultural -interpretation at the inner of the interactive communities, allowing to explain the events of the educative process, in where the speech had a fundamental paper, since permitted investigate about the formation of the professional and identify situations that received meaning in the pedagogical practices.

\section{Key Words:}

Curriculum, pedagogical Practice, professional competition, pedagogical model and periodic Table.

\section{INTRODUCCIÓN}

La profesión docente constituye aspectos relacionados con la función de enseñar del sujeto actuante en un contexto de aprendizaje. Un buen docente en cualquier área del conocimiento específico propone diferencias ideológicas frente a los demás; orienta la búsqueda del quehacer pedagógico; sabe motivar y confiar un espacio de acercamiento y diálogo. En consecuencia, puede decirse que la docencia de la Química es el proceso de enseñanza de unos saberes específicos, que requieren de preparación y planificación, y es aquí, donde la investigación es un elemento importante como fuente de enriquecimiento para la práctica docente.

Y es esta la razón, que nos motivo a realizar un análisis de los procesos de enseñanza, llevạdos a cabo en las Instituciones Educativas Distritales por los egresados en ejercicio de la Licenciatura en Química de la Universidad Distrital. 


\section{FUNDAMENTACION TEORICA}

Los cinco aspectos rëlevantes de este artículo son: currículo, prácticas pedagógicas, modelos pedagógicos, competencia profesional y tabla periódica en contexto etnográfico.

\subsection{Currículo}

Se ha señalado el papel decisivo que juega el currículo como herramienta en el proceso de enseñanza-aprendizaje, su importancia en la práctica del docente y su apoyo de la teoría pedagógica al aula.

En palabras de Stenhouse, "el currículo es un curso de acción, un objeto de acción simbólico y significativo para nuestros alumnos, el currículo es siempre hipotético cada vez necesita ser comprobado en su contenido no en el hecho de repetir conceptos, sino de desarrollar procesos de construcción por parte de profesores y estudiantes,s. Dicho de otro modo pretende establecer el perfil académico del estudiante en su formación inicial y el perfeccionamiento del docente.

Según Kemmis, "el curriculo pretende formar un hombre no solo en la teoría ni solo en la práctica, sino en la relación dialéctica entre ambàs, el currículo se configura entonces desde el exterior hacia el interior de las personas, en el análisis de la sociedad y la cultura detectando símbolos, mitos, lenguajes, valores, formas de producción y relación social para transformar la educación, is. En otras palabras el currículo busca construir el perfil integral del estudiante durante su formación, a través de un análisis cultural, de una planificación situacional, de una descentralización educacional, de una profunda investigación en el aula, brota como una alternativa de poder de participación y de emancipación social.

Según Magendzo, propone un currículo comprensivo como aquel que traduce formas para enfrentar la propia vida, aquel que esta en un proceso de búsqueda de negociación, de valoración, de crecimiento y de confrontación entre la cultura universal y la cultura de la cotidianidad ${ }^{7}$. Es decir se trata de formular un currículo pertinente en torno a la capacidad para resólver problemas, para aprender en colectividad rumbo a una cultura de integración llegando a la renovación de la institución escolar en general y el proyecto de innovación del centro educativo, y en concordancia con Bermúdez quien indica, un currículo pertinente es el que además de pedagogía conoce los intereses y necesidades de sus alumnos y por ende la comunidad sociocultural a la que pertenecen ${ }^{8}$.

\subsection{La práctica pedagógica, quehacer fundamental del educador con la comunidad}

La concepción de práctica docente varía de acuerdo a los autores en cuanto a los contextos de àprendizaje, tendencias sociales y'de poder, que permiten encontrar diversidades de procesos desarrollados y asumidos al interior del aula de clase.

\footnotetext{
Stenhouse. L. Investigación y desarrollos del currículo. Editorial Morato. Madrid: 1984, p. 62 Kemmis, S. Currículum: más allá de la teoría de la reproducción. Editorial Morato. Madrid: 1986. p. 143. Magendzo, A. Currículum y cultura en América Latina. Santiago de Cali: PIIE, 1991.

Bermúdez, Roberto. Otros. Diagnóstico sobre la Formación inicial y Permanente del Profesorado de Ciencias y Matemáticas en los Países Iberoamericanos. Ministerio de Educación y Ciencia. Editorial Mc Graw Hill. Madrid: 1994, p. 152-160.
} 
Según Bermúdez: "las prácticas pedagógicas en Ouímica están directamente relacionadas con la docencia y ésta se orienta alrededor de conceptos tales como: 1)es una actividad organizada, planeada y sistematizada que aborda las temáticas comprendidas en el currículo, 2) es la conjunción de la teóría y la práctica, 31 es la actividad académica que desarrollan los profesores, es la forma de trasmitir un currículo, 4) es la actividady asesoría del proceso enseñanza y aprendizaje, 5l es unà actividad profesional consistente en orientar y participar en la construcción de aprendizajes significativos, 6/ es la aplicación de un plan de estudios, 7) es un proceso de interacción entre profesores y alumnos con la finalidad de lograr aprendizajess". Lo anterior tiene sentido sí se ubican en la lógica de concepción y construcción de la práctica pedagógica de cada profesor. Todos los conceptos vertidos sobre la práctica pedagógica en Química como ejercicio de la docencia son válidos; considerando que la docencia es conducción, asesoría, orientación, aplicación, interacción, transmisión y participación. Además, es importante destacar que la práctica pedagógica implica conocer las políticas educativas, normas y los valores institucionales vigentes para que la función docente pueda desarrollarse en un marco de reflexión y producción permanente ${ }^{10}$. Es decir, que se mira el proceso y el contenido de lo que ocurre dentro de la escuela en términos de la estructura interna.

Bernstein distingue dos modalidades dè̃ prácticas pedagógicas; las visibles y las invisibles. En las prácticas pedagógicas visibles se reclama para sí una orientación y legitimación derivadas de la supuesta autonomía del saber, y las pedagogías invisibles afectan al principio de recontextualización inherente a la creación y sistematización de los contenidos, dando origen a diferentes modalidades de práctica pedagógica (progresistas, conservadoras y radicales).

\subsection{Modelo pedagógico}

Un modelo exige tomar una postura determinada, delimitando aspectos esenciales, propósitos, contenidos, secuencias y brindar finalmente una serie de herramientas para que estos puedan ser llevados a la práctica educativa.

En didáctica la palabra modelo aparece como muestra o estereotipo de posible alternativa de enseñanza-aprendizaje. "El modelo es un ésquema mediador entre la realidad y el pensamiento:"

En palabras de Porlan, un modelo pedagógico puede considerarse como un dispositivo de transmisión cultural que se deriva de una forma particular de selección, organización, transmisión y evaluación del conocimiento escolar. En esta dimensión que podemos llamar instruccional, el modelo está constituido por tres sistemas de mensajes: el currículo, la pedagogía y la evaluación ${ }^{12}$.

Según el tipo de experiencias en que se halla participado y su grado de interiorización las practicas de los docentes y el modelo pedagógico se pueden inscribir en alguna de las grandes corrientes pedagógicas: tradicional, romántico, constructivista y social.

Ibíd. p. 154

10 Ibíd. p. 160

1 FERNANDEZ, J. Y ElORTEGUI, N. Qué Piensan los Profesores Acerca de Cómo se Debe Enseñar. En: Enseñanza de las Ciencias. 1996. p-332.

i2 PORLAN, Rafael. Conocimiento de los Profesores una Propuesta Formativa en el Área de Ciencias. Colección investigación y enseñanza. Serie Fundamentos, volumen: 8. España: 1998, p.58. 
El modelo tradicional enfatiza una formación moldeadora en los estudiantes partiendo de la voluntad, la virtud, el rigor de la disciplina científica. El aprendizaje es el academicista, verbalista, en el que predomina una concepción rígida y estática en referencia al conocimiento escolar y a los cambios hacia otros enfoques ${ }^{13}$.

El modelo pedȧgógico romántico ${ }^{14}$ sostiene que el eje de la educación es lo que procede del interior del estudiante. El ambiente pedagógico debe ser el mas flexible posible para que el niño despliegue su interioridad, sus cualidades y habilidades naturales. El desarrollo natural del estudiante se convierte en la meta y a la vez el método de la educación. El docente debe convertirse en un auxiliar o amigo de la expresión libre, original y espontánea de los estudiantes.

El modelo pedagógico progresista o constructivista ${ }^{15}$ sostiene que la meta educativa es que cada individuo acceda, progresiva y secuencialmente a la etapa superior de su desarrollo intelectual, de acuerdo con las necesidades y condiciones de cada uno. El docente debe ser facilitador del acceso a estructuras cognitivas superiores que contribuyan al afianzamiento y desarrollo de sus estructuras mentales.

El modelo pedagógico social ${ }^{16}$ propone el desarrollo máximo y multifacético de las capacidades e intereses del estudiante. Tal desarrollo esta determinado por la sociedad, por la colectividad en la cual el trabajo productivo y la educación están íntimamente ligados para garantizar no solo el desarrollo del espíritu colectivo sino el conocimiento científico - técnico y el fundamento de la práctica para la formación científica de las nuevas generaciones.

Podríamos concluir entonces que el modelo pedagógico es una representación teórica de un sistema determinado que abarca conocimientos, métodos de enseñanza aprendizaje, teorías cognitivas y relación docente - estudiante, desplegados en la práctica pedagógica.

\subsection{Competencia - Competencia profesional}

La competencia es vista como una potencialidad o una capacidad para poner en escena una situación problemática y resolverla, para explicar su solución, para controlar y posiciònarse de ésta. La competencia sólo es visible a través de desempeños ${ }^{17}$. 18 y se manifiesta en los recursos con que cuenta un individuo para realizar una tarea o utilidad ${ }^{19}$

is Flórez, Rafael. Hacia una pedagogía del conocimiento (1994). Evaluación Pedagógica: cognición. Editorial Mc Graw Hill. Bogotá: 1999,'p. 33.

i4 Flórez, Rafael. Hacia una pedagogía del conocimiento (1994: p.168). Evaluación Pedagógica: cognición. Editorial Mc Graw Hill. Bogota: 1999, p. 37.

is Dewey, Piaget y Kolnberg. Citado por Florez, Rafael. Evaluación Pedagógica: cognición. Editorial MC Graw Hill. Bogota: 1999, p. 42- 43.

16 Flórez, Rafael. Hacia una pedagogía del conocimiento (1994: p.170). Evaluación Pedagógica: cognición. Editorial Mc Graw Hill. Bogota: 1999, p. 50.

17 Bogoya, Daniel. Proyecto sobre evaluación de competencias. Universidad Nacional de Colombia. Memorias del Taller sobre evaluación de Competencias básicas. Bogotá: 1999, p. 12.

18 Torrado, María. El desarrollo de las competencias: una propuesta para la educación colombiana. Universidad Nacional de Colombia. Memorias del Taller sobre evaluación de competencias básicas. Bogotá: 1999, p.15.

19 Cárdenas, F. Conocimientos, logros, habilidades, competencias y ... Lqué evaluar? Universidad Nacional de Colombia. Memorias del Taller sobre evaluación de competencias básicas. Bogotá: 1999, p. 20. 
Además, implica una comprensión de los temas con una clara significación y un sentido para el niño y el adolescente. Se busca un conocimiento situado más allá de un mero requerimiento curricular. Un conocimiento que se integre a las vivencias del estudiante, a su manera de estar en el mundo.. ${ }^{20}$

Para Chevez la competencia conlleva al resultado de un proceso de integración de habilidades y de conocimientos (saber, saber-hacer, saber-ser, saber-emprender... ) $^{21}$.

Hablar de competencias profesionales en educación no se trata sólo de saberes teóricos adquiridos sino de actuaciones (capacidades de actuación) que permitan a la persona ser capaz de reaccionar y tomar decisiones. Por esta razón, un profesional competente debe ser capaz de: identificar los obstáculos o los problemas, prever, elegir, planificar y poner en marcha la mejor estrategia, sacar conclusiones y aprendizajes para una nueva situación.

Hemos ya señalado el papel decisivo que juegan las competencias profesionales en la construcción del conocimiento y la importancia de su utilización por el docente, es por esto que se conceptualiza como un proceso de integración de habilidades y de conocimientos (saber, saber-hacer, saber-ser, saber-emprender...) en situaciones autenticas "contextos".

\subsection{Tabla Periódica}

Mendeleyev definió la tabla periódica como la organización de los elementos según los valores crecientes de sus pesos atómicos en series verticales, de modo que sus filas horizontales contienen elementos semejantes en sus propiedades; que también están organizados en orden creciente de sus pesos atómicos.

Por esta razón, la tabla periódica se considera un instrumento donde están organizados en hileras y columnas los diferentes elementos químicos conocidos, basados en la ley periódica, lo que ha permitido a la humanidad conocer el interior de la materia y ver aplicaciones de la química.

La ley periódica explica la variación periódica de las propiedades de los elementos químicos en función del peso atómico, lo que ha permitido encontrar nuevos elementos. ${ }^{22}$.

Una de las grandes virtudes del sistema periódico es quie permite estudiar comparativamente una serie de propiedades de los elementos, que reciben el nombre de propiedades periódicas, entre las que se encuentran: volumen atómico, radio atómico, radio iónico, potencial de ionización, afinidad electrónica y electronegatividad ${ }^{23}$.

20 Vinent, M. Lenguaje y competencias. Universidad Nacional de Colombia. Memorias del Taller sobre evaluación de competencias básicas. Bogotá: 1999, p. 35.

21 Chévez, $M$ : Las competencias en la educación para el trabajo. Seminario sobre formación profesional y empleo. Encuentro Iberoamericano de responsables de la formación profesional. México: 1998, p.1.

22 Villaveces, José. La Tabla Periódica. Un microscopio para ver el interior del átomo. COOTEC. Universidad Nacional de Colombia. Bogotá: 1994, p. 105

23 Jensen, W. Electronegativity from Avogadro to Pauling. Journal of Chemical Education. Volumen 80 No.3. 2003: $279-287$ 
Tal como lo cita José Luis Villaveces "pocos logran explicar porque la Ley Periódica fue considerada como uno de los triunfos más importantes del siglo XIX. Al fin y al cabo la construcción de un índice o de un manual es una labor paciente y ordenada pero bastante trivial y no debería haber bastado para dar fama internacional a Dimitri Ivanovich Mendeleyevi ${ }^{24}$.

En tal sentido consideramos la tabla periódica como un modelo organizado que interrelaciona los elementos en grupos y periodos, dependiendo de sus propiedades físicas como temperatura de fusión, temperatura de ebullición entre otras y de sus propiedades químicas reflejo de la ley periódica como electronegatividad, potencial de ionización.... con el fin de describir la materia, los cambios físicos y químicos y las variaciones de energía que se derivan de ellos.

\section{METODOLOGÍA}

El diseño metodológico se fundamenta en la etnografía porque propicia la interpretación cultural que funciona al interior de las comunidades en interacción; convirtiéndose ésta en tramas de significación. La interpretación se convierte en una red de significados y sentidos en la cual la validez se legitima de acuerdo a las condiciones observadas, porque no se desliga de los contextos particulares y específicos visualizando de este modo la interpretación de la cultura.

La "descripción densà" a la que se refiere Geertz, plantea las visiones y las funciones de la antropología en dirección de la interpretación, resaltando el papel de la etnografía como un método para las interpretaciones de sistemas culturales, textuales y narrativos, donde la cultura es un texto público en la cual la sociedad se puede redimir a través del mismo, la representación social pasa por el texto, los problemas sociales se convierten en textos, de los cuales se puede hacer lectura ${ }^{25}$.

La etnografía se caracteriza por ser interpretativa, analizar discursos sociales y procurar en la interpretación, rescatar "lo dicho»; lo que presta especial atención en desentrañar estructuras nimias de textos específicos, en ese sentido, los hallazgos etnográficos son particulares; de ahí, que las conclusiones del etnógrafo deben ser de carácter específico y circunstanciado. ${ }^{26}$

Por ende, las características de la interpretación cultural, reflejan mayor dificultad en el desarrollo teórico, que en otras disciplinas y otros métodos de investigación, debido precisamente a que no es predictiva, no se puede generalizar sobre casos particulares y limitarlas a una muestra mínima.

De acuerdo con los anteriores referentes epistemológicos etnográficos se consideró el siguiente diseño metodológico para los instrumentos:

Ob. Cit. Villaveces. La Tabla Periódica ........ 1994. p. 109.

25 Geertz, Clifford Interpretación de las culturas. Editorial Gedisa. Barcelona: 1987, p. 18

26 Ibíd.. p. 31. 
El trabajo de campo tuvo como técnica fundamental la observación, siendo ésta el mecanismo por excelencia para dilucidar los acontecimientos inscritos en el entramado de las prácticas pedagógicas realizadas por los egresados en ejercicio de Licenciatura en Química de la Universidad Distrital, de los cuales se analizo la información. En el proceso de inmersión en el ambiente escolar de esta población, el discurso tuvo un papel fundamental, puesto que a través de éste, se pudo indagar acerca de las diferentes formaciones iniciales de los egresados, así como se pudo instaurar conversaciones a través de las entrevistas que permitieron identificar situaciones que cobraron significado para unos y otros, en lo que se refiere a las prácticas pedagógicas.

Teniendo en cuenta el objeto de estudio, se diseño un sistema conceptual que se desprende del marco teórico y de la interacción realizada en las prácticas pedagógicas. De acuerdo con esto, se plasmaron conceptos propios del grupo de investigación en torno a los ejes de análisis, que permitió el diseño de los instrumentos y el desarrollo de la metodología distribuida en las siguientes fases:

En la primera fase de exploración y/o preparación se realizaron diferentes actividades con el fin de interiorizar conceptos teóricos: capacitación de docentes - estudiantes, concreción de la muestra, conversatorios, seminarios permanentes y actividades de campo.

La muestra de docentes obedecieron a los siguientes criterios: ser egresado del Proyecto Curricular de Licenciatura en Química de la Universidad Distrital, estar trabajando en el área de química inorgánica en un establecimiento público que perteneciera al Distrito con estudiantes del grado décimo y estrato socioeconómico 1,2 y 3 . Adicionalmente se encontraron las siguientes características: titulados hace más de cinco años, vinculados a la planta docente de la Secretaria de Educación del D.C., experiencia profesional entre cinco y veinte años y estudios postgraduales, concluidos o en curso.

La segunda fase correspondió al trabajo de campo y la recopilación de la información acerca de los discursos y las prácticas pedagógicas de los docentes. Se realizaron registros de video, se diseñaron y aplicaron entrevistas y encuestas a los docentes y a los estudiantes, y se construyeron los diarios de campo.

Para la tercera fase se procedió al análisis e interpretación de los datos, permitiendo la conceptualización de los temas centrales de esta investigación; además, permitió definir las categorías, para el análisis de documentos, registros de video, diarios de campo, la observación y las entrevistas.

El análisis de los documentos fue el instrumento que permitió la lectura de los aspectos que enmarcan los cuatro Proyectos Educativos Institucionales y el currículo de las instituciones educativas donde laboran los egresados.

Los registros de video de los diferentes docentes permitió ver la relación docentes estudiantes; el ambiente educativo; el conocimiento de la disciplina por parte de los profesores; las habilidades y competencias comunicativas; las estrategias de enseñanza; el dominio y claridad del docente acerca de la Tabla Periódica y su incidencia en el conocimiento de la disciplina (Química). 
Los diarios de campo registraron los comportamientos y actitudes relacionados con el quehacer y desempeño de los docentes y de los estudiantes de los cursos donde se produce el hecho educativo.

A través de la observación se pudo obtener información, con la cual se corroboró el contexto de análisis, en relación directa con roles y papeles, interacción docentesestudiantes, leriguajes empleados en el aula de clase, forma de construir los mensajes, desarrollo de estrategias didácticas, competencias por desarrollar, logros y demás situaciones que tienen que ver con el decir y el hacer en un acto concreto de educación escolar.

Con las entrevistas se indagó sobre las relaciones docentes - estudiantes, mediadas por los conceptos, comportamientos y acciones propias del acto pedagógico. Además, complementaron la información no registrada en los otros instrumentos, y facilitaron el acercamiento con los entrevistados para obtener información puntual, pertinente a la investigación.

El proceso de categorización se llevó a cabo de acuerdo con las variables, indicadores y datos obtenidos mediante la aplicación de los instrumentos y centrados directamente en la fase de la investigación puesta en marcha.

El nivel más importante en esta etapa lo constituye el análisis de los resultados, y favorecen la construcción de una matriz (anexo) para interpretar datos, compararlos, darles sentido y poner en evidencia las características relevantes, manifestadas por los docentes egresados del Proyecto Curricular de Licenciatura en Química, en los lugares donde ejercen la docencia.

\section{ANÁLISIS Y RESULTADOS}

La investigación etnográfica en la que se enmarca este artículo, contempla aspectos teóricos que contribuyen a establecer cinco categorías: PEl, práctica pedagógica, modelos pedagógico, competencia y tabla periódica, lo que permite organizar y analizar la información recolectada de las observaciones ordinarias y de los registros de video.

En la categoría correspondiente al PEI, se analizo el plan de estudios, el perfil del estudiante, la formación integral y los proyectos de innovación o apoyo; evidenciándose el cumplimiento de estos en los documentos institucionales. Sin embargo, al examinar el trabajo en el aula de los docentes observados, en relación con su estrategia pedagógica implementada, el plan de estudios que desarrolla, la ejecución de un proyecto de apoyo de acuerdo a lo establecido por la institución con el fin de alcanzar las competencias previstas y su perfil, nos encontramos con una situación por completo diferente que no se conjuga con ninguna de las subcategorías anteriores.

Desde esta perspectiva, son varias las causas por las cuales el Proyecto Educativo Institucional va por un lado y el trabajo de los docentes por otra; en primer lugar se advierte un cierto descuido de parte de las instituciones por la falta de control en la ejecución de las propuestas contempladas para hacer coherente el PEl con el trabajo de enseñanza que se adelanta en las aulas. 
Además, no existen planes de acción conjunta en el área de química para desarrollar proyectos de aula, tampoco seminarios de actualización permanentes apoyadas por la institución sobre nuevas tendencias de educación en ciencias; es por esto que algunos docentes adelantan cursos afines a su disciplina de manera autónoma-y otros acuden a talleres que la Institución Educativa brinda, pero que la mayoría de veces no se relacionan con su área de trabajo.

Por otra parte, los estudiantes se limitan, en la mayoría de los casos, simplemente a hacer acto de presencia, sin importarles las condiciones đel proceso educativo que se adelanta en la Institución, con el incumplimiento de los horarios de clase, con la escasa participación en la misma, y dedicarse a cuestiones diferentes mientras están recibiendo las instrucciones y enseñanzas del docente. Esto repercute en la misión que las Instituciones tienen como fundamento.

Con esta categoría se advierte un perfecto apoyo documental sobre la información del $\mathrm{PEl}$, pero ninguna aplicación real del mismo, debido a la falta de mecanismos adecuados de comunicación entre la Institución, los docentes y los estudiantes.

En cuanto a la categoría de práctica pedagógica, se analizo aspectos como: la preparación de clases, la metodología del proceso de enseñanza, la interacción estudiante - docente y el discurso del docente; lo que permitió conocer su desarrollo al interior del aula y arrojar los siguientes resultados:

De los cinco docentes tomados como muestra, sólo dos de ellos cumplen con la tarea de preparar, planear correctamente las clases, los otros dos lo hacen ocasionalmente y uno de ellos nunca. Esto se debe a una cierta tendencia por parte de los docentes con más antigüedad de confiar en la memoria y en los conocimientos que supuestamente han perfeccionado, develando una excesiva confianza en las estrategias que sigue para adelantar sus clases y el programa; por esta razón, los docentes demuestran dificultades y deficiencias conceptuales y pedagógicas en el proceso de enseñanza, en los temas relacionados con la tabla periódica, y en los estudiantes un conformismo, sin autocrítica para formular otros juicios de valor. Se requiere en este caso, un mayor control por parte de las instituciones para alcanzar los niveles deseados, un seguimiento en la preparación de las clases y adelantar un proceso de actualización adecuado y permanente de los educadores, con el fin de alcanzar los objetivos del PEI.

La metodología permitió analizar los procesos de enseñanza de tipo magistral, participativo, por proyectos, teóricos y experimentales, mostrando que los más utilizados en orden descendente son: el teórico, magistral, participativo, por proyectos y experimental. Esto se debe a que la mayoría de las clases son rutinarias, generando repetición mecánica e irreflexiva, control de la clase e impidiendo que los estudiantes tomen decisiones conscientemente, convirtiéndose de esta manera en un docente facilitador y no orientador del proceso de enseñanza - aprendizaje; además conduce a la pasividad del proceso, a frenar la creatividad y a prevalecer el modelo tradicional que se caracteriza por ser rígido, pudiendo ser un obstáculo para llevar a buen termino los deseos de innovación de los estudiantes.

En relación a la interrelación docente - estudiante que comprende la comunicación y el dominio del grupo en el desarrollo de la clase, prevalece el dialogo centrado en el saludo, 
ordenes, advertencias, observaciones y discusiones donde finalmente se impone el docente; por otro lado, el dominio del grupo se encuentra en equilibrio entre la flexibilidad y la rigidez, lo cual no indica a ciencia cierta cual es la inclinación en este caso, ya que depende de la manera como el docente asuma su posición frente a las acciones y situaciones con los estudiantes.

En cuanto al discurso del docente, prevalecen las disertaciones de tipo oral e informativo combinados con menos frecuencia los discursos cotidianos y científicos, y ocasionalmente los descriptivos, explicativos y argumentativos.

Este fenómeno influye en la toma de decisiones, teniendo en cuenta que dentro de las competencias básicas de todo profesional, cualquiera que sea el programa de estudio, está la de adquirir capacidades suficientes para rendir informes, escribir artículos, ensayos y ponencias.

En cuanto a la categoría de competencia se analizo la comunicativa y la cognoscitiva, arrojando una tendencia a fortalecer la interpretación, la argumentación y la proposición, por parte de la mayoría de los docentes; situación que muestra la falta de concreción del PEI por parte de los docentes en el desarrollo de cada uno de los programas. Frente a la cognoscitiva los docentes la favorecen pero no en su totalidad, lo que debería indicar una total responsabilidad de los docentes en el desarrollo de esta competencia, teniendo en cuenta el compromiso de la institución en lograr los más altos niveles en el proceso educativo.

En torno a la categoría modelo pedagógico, se enfatiza en el tradicional y en el constructivista, encontrándose un equilibrio de estos dos. Esto es consecuencia de factores incidentes a lo largo de la formación y experiencia profesional de los docentes, al estar en contacto con uno o varios de estos modelos (Pórlan, 1994). Como lo cita Bojacá (2002), los docentes tienen una amalgama de modelos adoptados de su experiencia profesional como aprendices, y de las oportunidades que brinda el sistema educativo, los cuales determinan las características de sus teorías implícitas sobre la enseñanza.

En la categoría tabla periódica se indago sobre la epistemología, la secuenciación, el manejo y profundización durante el desarrollo de este tema en particular, evidenciando que los docentes enfatizan en estos aspectos, sin embargo rara vez o nunca los utilizan en el desarrollo de los demás contenidos del programa. Por esta razón, se debería implementar y fortalecer estos tres campos durante el proceso académico, ya que el tema de la tabla periódica es empleado como eje transversal de la química.

\section{CONCLUSIONES}

La práctica pedagógica en Química debe lograr que el docente en ejercicio se apropie de la realidad y la proyecte en calidad de objeto y sujeto de su propio pro-ceso, con esto, el estudiante deja de ser un simple objeto, un simple receptor de la información, ya que se evidencio a través de los instrumentos lo que afirma Villaveces (1994), que los estudiantes cuando se enfrentan por primera vez a la Tabla Periódica no interpretan en ella su periodicidad química, puesto que casi todos tienen la idea que es una tabla 
con información sobre los elementos.

La práctica pedagógica en Química debe ser coherente y estar articulada con el currículo y con los Proyectos educativos institucionales, los cuales demarcan el horizonte educativo. Por tanto, para mejorar las prácticas pedagógicas en esta área, las instituciones les corresponden considerar la necesidad de capacitación y actualización de los profesores', en el ámbito de sus competencias profesionales, y en el dominio teórico y conceptual de la Ouímica.

La escuela actual no responde a las expectativas, aún subyacen las raíces del aprendizaje de las informaciones específicas y aisladas que en ningún momento favorecen las relaciones conceptuales y menos aún el acercamiento a la interdisciplinariedad; esto se debe a una cierta tendencia por parte de los docentes con más antigüedad de confiar en la memoria y en los conocimientos que supuestamente han perfeccionado, develando una excesiva confianza en las estrategias que sigue para adelantar sus clases y el programa; por esta razón, los docentes demuestran dificultades y deficiencias conceptuales y pedagógicas en el proceso de enseñanza, en los temas relacionados con la tabla periódica, y en los estudiantes un conformismo, sin àutocrítica para formular otros juicios de valor.

No se evidencio como los cursos de formación posgradual han repercutido en la creación e innovación de la enseñanza de la química, debido a la frágil infraestructura de las instituciones que no facilitan la calidad en los procesos de actualización docente, igualmente se podría ingerir que falta iniciativa propia de los docentes para la innovación, actitud positiva a la investigación, y descubridor de potencialidades.

\section{BIBLIOGRAFÍA}

BERMÚDEZ, R. Otros. Diagnóstico sobre la Formación Inicial y Permanente del Profesorado de Ciencias y Matemáticas en los Países Iberoamericanos. Ministerio de Educación y Ciencia. Editorial Mc Graw Hill. Madrid: 1994.

BOJACA, B. ¿Qué hacemos los maestros cuando hablamos en el aula? Concepciones sobre la enseñanza de.la lengua. Universidad Distrital: Bogotá: 2002.

BOGOYA, D. Proyecto sobre evaluación de competencias. Universidad Nacional de Colombia. Memorias del Taller sobre evaluación de Competencias básicas. Bogotá: 1999.

CÁRDENAS, F. Conocimientos, logros, habilidades, competencias y... Lqué evaluar? Universidad Nacional de Colombia. Memorias del Taller sobre evaluación de competencias básicas. Bogotá: 1999.

CHÉVEZ, M. Las competencias en la educación para el trabajo. Seminario sobre formación profesional y empleo. Encuentro Iberoamericano de responsables de la formación profesional. México: 1998.

FERNÁNDEZ, J y Elortegui, E. Qué piensan los profesores acerca de cómo se debe enseñar. En: Investigación y Experiencias didácticas. Bogotá.1996.

FLOREZ, R. Evaluación Pedagógica: cognición. Editorial Mc Graw Hill. Bogota: 1999. 
FLOREZ, R. Hacia una pedagogía del conocimiento. Editorial MC Graw Hill. Bogotá: 1994.

GEERTZ, C Interpretación de las culturas. Editorial Gedisa. Barcelona: 1987.

JENSEN, W. Electronegativity from Avogadro to Pauling origins of the Electronegativity concept. Journal of Chemical Education. Volumen 73. 1996.

JENSEN, W. Electronegativity from Avogadro to Pauling. Journal of Chemical Education. Volumen 80 No.3. 2003.

KATZ, J. Química y Reactividad Química. Editorial Thomson. México: 2003.

KEMMIS, S. Currículum: más allá de la teoría de la reproducción. Editorial Morato. Madrid: 1986.

MAGENAZO, A. Currículum y cultura en Américá Latina. Santiago de Cali: PliE, 1991.

PORLAN, R. Las concepciones epistemológicas de los profesores: el caso de los estudiantes del magisterio. Investigación en la escuela. España: 1994.

PORLAN, R. Conocimiento de los Profesores una Propuesta Formativa en el Área de Ciencias. Colección investigación y enseñanza. Serie Fundamentos, volumen: 8 . España: 1998.

POZO. J. Aprender y Enseñar Ciencia: del conocimiento cotidiano al conocimiento científico. Ediciones Morata, Madrid: 1998.

POZO, M. Teorías cognitivas del aprendizaje. Editorial Morata. Madrid: 1989.

QUINTANA, J. Plan de estudios fundamentado en competencias: no es para la escuela, es para la vida. Bogotá: 1999.

STENHOUSE, L. Investigación y desarrollos del currículo. Editorial Morato. Madrid: 1984.

TORŔRAO, M. El desarrollo de las competencias: una propuesta para la educación colombiana. Universidad Nacional de Colombia. Memorias del Taller sobre evaluación de competencias básicas. Bogotá: 1999.

VINENT, M. Lenguaje y competencias. Universidad Nacional de Colombia. Memorias del Taller sobre evaluación de competencias básicas. Bogotá: 1999.

VILLAVECES, J. La Tabla Periódica. Un microscopio para ver el interior del átomo. COOTEC. Universidad Nacional de Colombia. Bogotá: 1994. 
ANEXO

CUADRO DE CATEGORIZACIÓN

\begin{tabular}{|c|c|c|c|c|c|c|c|c|}
\hline \multirow{2}{*}{ CATEGORÍA } & \multirow{2}{*}{\multicolumn{3}{|c|}{ SUBCATEGORÍA }} & \multicolumn{5}{|c|}{ INSTITUCION } \\
\hline & & & & A & $\mathrm{B}$ & & & D \\
\hline \multirow{5}{*}{$\begin{array}{l}\text { PROYECTO } \\
\text { EDUCATIVO } \\
\text { INSTITUCIONAL }\end{array}$} & \multicolumn{3}{|l|}{ Currículo } & 1 & 1 & & & 1 \\
\hline & \multicolumn{3}{|l|}{ Plan de estudios } & 1 & 1 & & & 1 \\
\hline & \multicolumn{3}{|c|}{ Perfil del estudiante } & 1 & 1 & & & 1 \\
\hline & \multicolumn{3}{|c|}{ Formación Integral } & 1 & 1 & & & 1 \\
\hline & \multicolumn{3}{|c|}{ Proyectos de Innovación o Apoyo } & 1 & 1 & & & 1 \\
\hline \multirow{2}{*}{ CATEGORÍA } & \multirow{2}{*}{\multicolumn{3}{|c|}{ SUBCATEGORIA }} & \multicolumn{5}{|c|}{ DOCENTES } \\
\hline & & & & $\overline{\mathrm{A} 1}$ & $\mathrm{~B} 1$. & $\overline{B 2}$ & $\mathrm{C} 1$ & D1 \\
\hline \multirow{19}{*}{$\begin{array}{c}\text { PRÁCTICA } \\
\text { PEDAGÓGICA }\end{array}$} & \multirow{6}{*}{$\begin{array}{l}\text { Preparación de c } \\
\text { Metodología } \\
\text { del proceso } \\
\text { enseñanza }\end{array}$} & lase & & 3 & 1 & 2 & 1 & 2 \\
\hline & & Magistral & & 2 & 1 & 1 & 1 & 2 \\
\hline & & Participativa & & 3 & 2 & 3 & 1 & 1 \\
\hline & & Proyectos & & 3 & 3 & 3 & 1 & 3 \\
\hline & & Teórico & & 1 & 1 & 1 & 2 & 1 \\
\hline & & Experimental & & 2 & 3 & 2 & 3 & 3 \\
\hline & \multirow{4}{*}{$\begin{array}{l}\text { Interrelación } \\
\text { Docente- } \\
\text { Estudiante }\end{array}$} & \multirow{2}{*}{ Comunicación } & Dialogo & 3 & 1 & 3 & 1 & 1 \\
\hline & & & Monologo & 1 & 2 & 1 & 3 & 3 \\
\hline & & \multirow{2}{*}{$\begin{array}{l}\text { Dominio del } \\
\text { grupo }\end{array}$} & Flexible & 1 & 3 & 3 & 2 & 1 \\
\hline & & & Rígido. & 3 & 1 & 1 & 3 & 3 \\
\hline & \multirow{9}{*}{$\begin{array}{l}\text { Discurso del } \\
\text { docente }\end{array}$} & Cotidiano & & 1 & 2 & 1 & 2 & 1 \\
\hline & & Científico & & 3 & 1 & 3 & 1 & 1 \\
\hline & & Escolar & & 2 & 2 & 2 & 1 & 1 \\
\hline & & Oral & & 1 & 1 & 1 & 1 & 1 \\
\hline & & \begin{tabular}{|l|} 
Escrito \\
\end{tabular} & & 3 & 1 & 3 & 3 & 3 \\
\hline & & Informativo & & 1 & 1 & 1 & 1 & 1 \\
\hline & & Descriptivo & & 3 & 1 & 3 & 1 & 1 \\
\hline & & Explicativo & & 3 & 1 & 3 & 1 & 1 \\
\hline & & Argumentativo & & 3 & 1 & 3 & 1 & 1 \\
\hline \multirow{4}{*}{ COMPETENCIA } & \multirow{3}{*}{ Comunicativa } & Interpretativa & & 3 & 1 & 3 & 1 & 1 \\
\hline & & Arqumentativa & & 3 & 1 & 3 & 1 & 1 \\
\hline & & Propositiva & & 3 & 1 & 3 & 1 & 1 \\
\hline & \multicolumn{3}{|l|}{ Cognoscitiva } & 2 & 1 & 2 & 1 & 1 \\
\hline \multirow{2}{*}{$\begin{array}{c}\text { MODELO } \\
\text { PEDAGÓGICO }\end{array}$} & \multicolumn{3}{|l|}{ Tradicional } & 1 & 2 & 1 & 3 & 3 \\
\hline & \multicolumn{3}{|l|}{ Constructivista } & 3 & 2 & 3 & 1 & 1 \\
\hline \multirow{3}{*}{$\begin{array}{c}\text { TABLA } \\
\text { PERIÓDICA }\end{array}$} & \multicolumn{3}{|c|}{ Base epistemológica } & 2 & 1 & 3. & 1 & 1 \\
\hline & Secuenciación & & & 2 & 1 & 3 & 1 & 1 \\
\hline & Manejo y profun & Jización & & 2 & 1 & 2 & 1 & 1 \\
\hline
\end{tabular}

Escala valorativa: 1: Siempre 2: Ocasionalmente $3:$ Nunca

Interpretando siempre, en el sentido, que se hizo presente el aspecto, la subcategoría, en el contexto, más no que cumple satisfactoriamente la acción o el propósito.

Ocasionaimente, se evidencia periódicamente la subcategoría, pero no significa que la acción realizada se cumpla satisfactoriamente

Nunca, hace referencia a la ausencia de una subcategoría en el desarrollo del proceso, incidiendo sobre la calidad de la acción observada. 